\title{
TUNNEL CURRENT FEATURES CAUSED BY DEFECT ASSISTED PROCESS IN RESONANT-TUNNELLING STRUCTURES
}

\author{
A.E. Belyaev, S.A. Vitusevich, B.A. Glavin, R.V. Konakova \\ Institute of Semiconductor Physics, National Academy of Sciences of Ukraine \\ Kiev 252028, Ukraine
}

W. Dobrowolski, A. Mąkosa

Institute of Physics, Polish Academy of Sciences

Al. Lotników 32/46, 02-668 Warszawa, Poland

L.N. Kravchenko and E.S. Gornev

Science and Research Institute of Molecular Electronics

Zelenograd, Moscow district, Russia

\begin{abstract}
An extra channel of electron tunnelling through a double-barrier resonant-tunnelling GaAs/AlGaAs heterostructure caused by impurity assisted tunnelling was identified. We argue that it is due to DX centres associated with dopant donor atoms which diffused into the AlGaAs barrier layer.
\end{abstract}

PACS numbers: 73.40.Gk, 85.30.Mn

Practically, all resonant-tunnelling devices contain as their important part an undoped spacer layer separating a heavily-doped layer from the barrier. Including the spacer layer into the structure has several good points. It permits, for instance, to decrease the device capacitance, to prevent impurity diffusion into the active part of the device, to eliminate an effect of the long-range Coulomb potential on the tunnelling, and thus, to improve the current-voltage characteristics of the device. However, a thick spacer layer is also a drawback which can essentially affect the transport properties in the injector. Actually, owing to a light doping in a close vicinity of the emitter barrier, an applied bias voltage creates an accumulation layer. Generally, the presence of the accumulation layer makes the tunnelling dynamics worse, because the recharging processes which occur in this layer are slow as compared with the tunnelling time thus giving rise to an extra frequency cut-off. The physical processes occurring in the injector part of device are still of interest as being of a large significance for the device operation.

In this work we grew a structure whose composition was chosen so as to obtain the flat band regime in the emitier spacer under resonance condition. High 


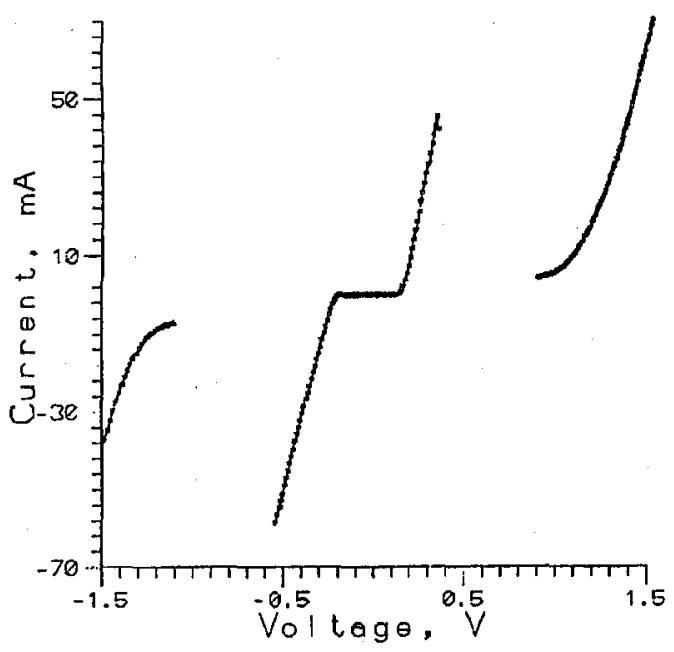

Fig. 1. Current-voltage characteristic of double-barrier heterostructure at $4.2 \mathrm{~K}$.

quality $\mathrm{Al}_{0.3} \mathrm{Ga}_{0.7} \mathrm{As}(1.7 \mathrm{~nm})-\mathrm{GaAs}(5.6 \mathrm{~nm})-\mathrm{Al}_{0.3} \mathrm{Ga}_{0.7} \mathrm{As}(1.7 \mathrm{~nm})$ double-barrier resonant-tunnelling diodes with $n^{+}$-graded $n-p^{-}$emitter and collector regions were fabricated. At room temperature they displayed the peak current density $8 \times 10^{3} \mathrm{~A} / \mathrm{cm}^{2}$ and the peak-to-valley ratio 3 . At $4.2 \mathrm{~K}$ the respective values were $1.6 \times 10^{4} \mathrm{~A} / \mathrm{cm}^{2}$ and 10 (Fig. 1). The dopant impurity (Si) concentration in $n^{+}$ layers was $3 \times 10^{18} \mathrm{~cm}^{-3}$ and varied smoothly down to $10^{17} \mathrm{~cm}^{-3}$ in the graded $n$ layers. The width of the $p^{-}$layers adjacent to the barriers was $1.5 \mathrm{~nm}$ on one side and $14 \mathrm{~nm}$ on the other. Two quasi-bound states are expected to appear in this structure, with energy of $70 \mathrm{meV}$ and $260 \mathrm{meV}$, but actually only one resonance peak was observed under both bias polarities.

We also observed a small bump in the differential conductance measured as a function of the applied voltage in front of the principal resonance peak (Fig. 2). This feature appeared under the reverse bias only, i.e. when electrons were injected from the thin-spacer side. Magnitude of this bump increases as a function of the magnetic field applied parallel to the current direction up to a field of $8 \mathrm{~T}$ (Fig. 3). Further increase in the magnetic field leads to a decrease in the bump amplitude.

We suppose that the above feature of the tunnel current is the manifestation of an additional resonant-tunnelling channel via the bound state of a donor atom located in the barrier. Actually, due to a small width of $p^{-}$layer adjacent to the barrier on one side of the structure, the dopant atoms whose concentration is at least $10^{17} \mathrm{~cm}^{-3}$ can diffuse into the barrier to create trap levels. There are two kinds of impurity-induced states which can be present in $\mathrm{Al}_{x} \mathrm{Ga}_{1-x} \mathrm{As}$ alloy. One of them is a shallow hydrogen-like state and the other is DX centre [1]. The first state has the binding energy of about (or smaller than) $7 \mathrm{meV}$ and its localization length is of the order of $10 \mathrm{~nm}$ for a direct-gap alloy. The second type of states has the binding energy of about $100 \mathrm{meV}$ for $x=0.3$ and its localization length is 


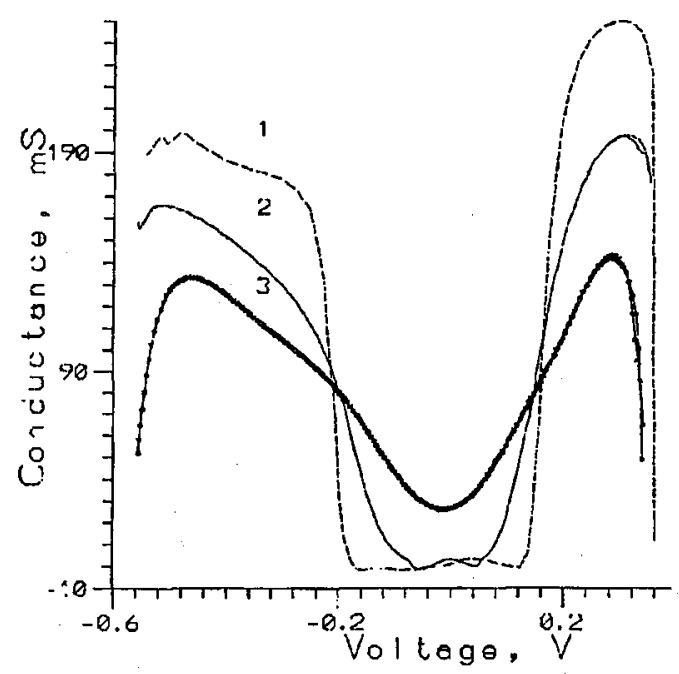

Fig. 2. Dependencies of differential conductivity on bias voltage, measured at temperatures $4.2 \mathrm{~K}(1), 110 \mathrm{~K}(2)$ and $300 \mathrm{~K}(3)$.

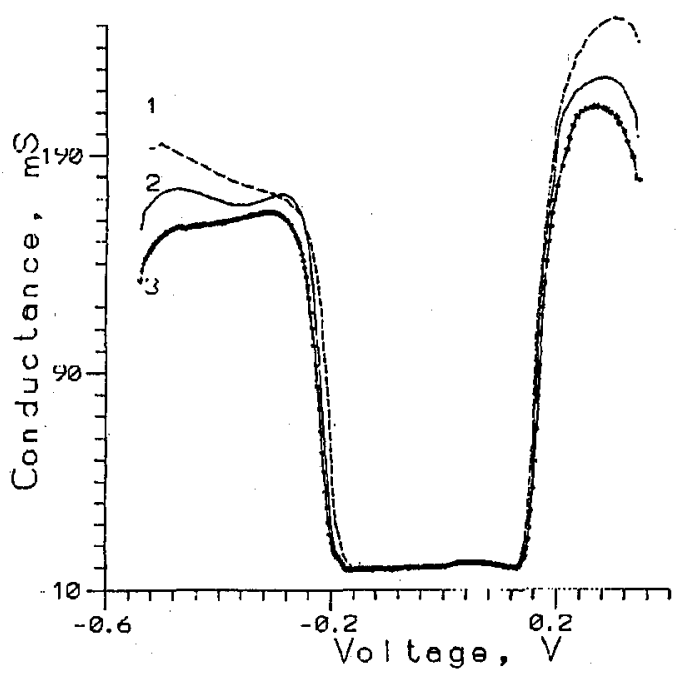

Fig. 3. Dependencies of differential conductance on the bias voltage in magnetic fields $1.0 \mathrm{~T}(1), 8 \mathrm{~T}(2), 13 \mathrm{~T}(3)$.

0.5-1 nm in bulk material. In our structures Si atoms cannot produce hydrogen-like states, because their localization length is considerably larger than the thickness of the barrier layers. Instead, the DX centres can appear in the barriers, since their localization length is small enough. Moreover, the $\mathrm{DX}^{-}$state is the lowest 
donor state for the alloy with $x=0.3$. Impurity atoms can also lead to additional quasi-bound states in the well. However, we can neglect their contribution to the tunnel current in the investigated structures because they are hidden inside a broad principal resonance level, so as the barriers have relatively low thickness and height.

Ilaving in mind the above considerations, we accept that the only impurity states, which can affect the current-voltage characteristics of the investigated diodes, are the $\mathrm{DX}$ centres in $\mathrm{Al}_{x} \mathrm{Ga}_{1-x} \mathrm{As}$ barriers. Tunnelling via the energy level associated with these defects gives rise to an additional contribution to the tunnel current [2]. On the other hand, the presence of charged centres in the barrier changes the potential profile of the structure [3]. The consequence of the latter is probably that only one resonance peak in the current-voltage curve is observed which is due to the tunnelling via the first excited quasi-bound state. Tunnelling through the ground quasi-bound state does not occur because its energy level is lowered below the Fermi energy in the emitter region. At zero bias voltage the first excited quasi-bound level in the well has energy $260 \mathrm{meV}$ and lies above the DX level. The latter will contribute to the tunnel current at such bias voltage at which the DX level in the barrier matches the quasi-bound level in the well. Obviously, in our structure this is possible only under reverse bias, i.e., when the electrons tunnel from the thin-spacer side and the DX centres are located in the emitter barrier. In this case the bias voltage shifts the quasi-bound level larger than the DX levels, so that the both coincide at certain bias voltage. Then an additional current flows as a result of the defect assisted tunnelling. In the case when the DX centres were located in the collector barrier, the bias voltage would shift the relevant levels almost equally so that they would never coincide. This is just what we observe in the experiment. Therefore, we attribute the bump appearing on the differential-conductance versus bias-voltage curve to the DX centre-assisted resonant tunnelling. Additional confirmation of such conclusion is the coincidence of the temperature at which the bump vanishes with the temperature at which the DX centres begin to be ionised [4].

In conclusion, we have presented experimental results showing the contribution of impurity states in the barrier to the resonant tunnelling. Analysis of these results allowed us to identify these states as connected with strongly localised DX centres.

\section{References}

[1] D.J. Chadi, J.K. Chang, Phys. Rev. B 39, 10063 (1989).

[2] D. Stievenard, X. Letarte, M. Lannoo, Appl. Plyys. Lett. 61, 1582 (1992).

[3] T.C.L.G. Sollner, H.Q. Le, C.A. Correa, W.D. Goodhue, Appl. Phys. Lett. 47, 36 (1985).

[4] A.E. Belyaev, Yu.S. Ryabchenko, M.K. Sheinkman, H.J. von Bardeleben, Semicond. Sci. Technol. 11, 68 (1996). 